\title{
Intra- and Inter-Rater Reliability of Ultrasound Measurements of Supraspinatus Tendon Thickness, Acromiohumeral Distance, and Occupation Ratio in Patients With Shoulder Impingement Syndrome
}

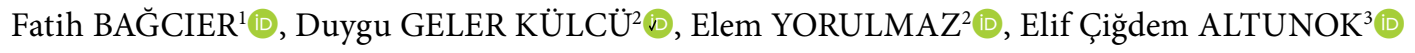 \\ ${ }^{1}$ Department of Physical Theraphy and Rehabilitation, Kars Harakani State Hospital, Kars, Turkey \\ ${ }^{2}$ Department of Physical Theraphy and Rehabilitation, Haydarpaşa Numune Training and Research Hospital, Istanbul, Turkey \\ ${ }^{3}$ Department of Biostatistics and Medical Informatics, Yeditepe University, Faculty of Medicine, Istanbul, Turkey
}

\begin{abstract}
Objectives: This study aims to evaluate the intra- and inter-rater reliability coefficients of the supraspinatus tendon thickness, acromiohumeral distance, and occupation ratio in patients with shoulder impingement syndrome.

Patients and methods: The study included 83 patients ( 21 males, 62 females; mean age $51.6 \pm 11.0$ years; range, 26 to 70 years) with shoulder impingement syndrome. The supraspinatus tendon thickness, acromiohumeral distance, and occupation ratio values were obtained one week apart by two observers. The intraclass correlation coefficient (ICC), minimum detectable change, and standard error of measurement were calculated.

Results: The first observer had excellent intra-rater reliability in all measurements (ICC $>0.90$ ) with minimum detectable change of $0.740-0.047 \mathrm{~mm}$ and standard error of measurement of $0.017-0.26 \mathrm{~mm}$. The second observer had excellent intra-rater reliability in supraspinatus tendon thickness and acromiohumeral distance measurements (ICC >0.90) with minimum detectable change of $0.498-0.770 \mathrm{~mm}$ and standard error of measurement of $0.18-0.28 \mathrm{~mm}$ and good intra-rater reliability in the occupation ratio measurement (ICC; $0.75-0.90)$ with minimum detectable change of $0.060 \mathrm{~mm}$ and standard error of measurement of $0.022 \mathrm{~mm}$. Inter-rater reliability coefficients were 0.916 (95\% confidence interval [Cl]; 0.873-0.945) for supraspinatus tendon thickness, $0.943(95 \% \mathrm{Cl} ; 0.914-0.963)$ for acromiohumeral distance with minimum detectable change of $0.673 \mathrm{~mm}$ and standard error of measurement of $0.243 \mathrm{~mm}$ and 0.790 (\%95 Cl; $0.693-0.853$ ) for occupation ratio with minimum detectable change of $0.077 \mathrm{~mm}$ and standard error of measurement of $0.028 \mathrm{~mm}$.

Conclusion: These findings suggest that ultrasonographic measurements of the supraspinatus tendon thickness, acromiohumeral distance and occupation ratio can be reliable and consistent for clinical evaluation of patients with shoulder impingement syndrome in terms of supporting diagnosis and monitoring the treatment effect.

Keywords: Reliability, shoulder impingement syndrome, ultrasound.
\end{abstract}

Shoulder impingement syndrome is one of the most common causes of shoulder pain. ${ }^{1}$ It was originally described as encroachment and mechanical compression of the subacromial soft tissues against the coracoacromial arch, typically during overhead arm elevation. ${ }^{2}$ Neer $^{2}$ described the compression of tendon structures under the acromion as external mechanisms and the presence of pathologies in the tendon itself as internal mechanisms. Patients with shoulder impingement syndrome have shoulder pain particularly during arm elevation which may be caused by a narrowing of acromiohumeral distance..$^{3,4}$ Provocative tests such as the Neer and Hawkins test aim to compress the soft tissue between the tuberculum major and the coracoacromial

Received: March 24, 2019 Accepted: November 10, 2019 Published online: February 07, 2020

Correspondence: Fatih Bağcıer, MD. Kars Harakani Devlet Hastanesi Fiziksel Tıp ve Rehabilitasyon Kliniği, 36200 Kars, Türkiye. Tel: +90 442 - 3447087 e-mail: bagcier_42@hotmail.com

\section{Citation:}

Bağcıer F, Geler Külcü D, Yorulmaz E, Altunok EÇ. Intra- and Inter-Rater Reliability of Ultrasound Measurements of Supraspinatus Tendon Thickness, Acromiohumeral Distance, and Occupation Ratio in Patients With Shoulder Impingement Syndrome. Arch Rheumatol 2020;35(3):385-393. 
arch, and thereby narrowing the acromiohumeral distance. Therefore, characterizing subacromial dimensions such as acromiohumeral distance and the supraspinatus tendon thickness may enhance the understanding of the underlying mechanisms of shoulder impingement syndrome and support the diagnosis. ${ }^{5,6}$ In recent years, ultrasonography has been widely used for the diagnosis of musculoskeletal pathologies because of its advantages such as non-invasiveness, broad accessibility, and easy applicability., ${ }^{7}, 8$ recent studies, ultrasonographic measurements have been found to be consistent with those of magnetic resonance imaging for determining the pathology of subacromial structures, including the supraspinatus tendon, subacromial bursa, and acromiohumeral region. ${ }^{9,10}$ The normal acromiohumeral distance, as measured by ultrasound in the neutral shoulder position, is reportedly $9-12 \mathrm{~mm}$, which is reduced to 6-10 $\mathrm{mm}$ in patients with varying degrees of tendon pathology. ${ }^{11-14}$ In patients with shoulder impingement syndrome, the supraspinatus tendon has been found to be thicker compared to asymptomatic shoulders; ${ }^{6,15-17}$ however, it was found thinner in another study. ${ }^{5}$ In a recent study, Michener et al. ${ }^{11}$ identified the relationship between supraspinatus tendon and the concurrent acromiohumeral distance, known as the occupation ratio. They hypothesized that the supraspinatus tendon would occupy a relatively greater proportion of the acromiohumeral distance in patients with shoulder impingement syndrome. ${ }^{11}$ Although there was no difference in acromiohumeral distance between the shoulder impingement syndrome and control groups, the supraspinatus tendon thickness and occupation ratio were significantly greater in the former. Hence, we also consider the occupation ratio as an important parameter of shoulder impingement syndrome that can be evaluated by ultrasonography. Although ultrasonography is easy to apply, the reliability of measurements must be confirmed. However, the majority of reliability assessments have been conducted on asymptomatic populations, and relatively few studies have evaluated the reliability of ultrasonographic measurements of the acromiohumeral distance and supraspinatus tendon thickness in patients with shoulder pain. ${ }^{4,15,16,18}$ To the best of our knowledge, the reliability of occupation ratio measurement has not yet been evaluated.
Therefore, in this study, we aimed to evaluate the intra- and inter-rater reliability coefficients of the supraspinatus tendon thickness, acromiohumeral distance, and occupation ratio in patients with shoulder impingement syndrome.

\section{PATIENTS AND METHODS}

This study was conducted at Haydarpaşa Numune Training and Research Hospital between August 2018 and November 2018. A total of 100 participants were initially enrolled who were admitted to our outpatient clinic complaining of shoulder pain. Then, due to nonattendance for second measurements, 17 patients were excluded; thus, only 83 patients (21 males, 62 females; mean age $51.6 \pm 11.0$ years; range, 26 to 70 years) were included for analysis. Patients were diagnosed with shoulder impingement syndrome according to the physical examination with at least three positive clinical tests out of five (Neer, Hawkins-Kennedy, painful arc, Jobe, and external rotation resistance). ${ }^{19}$ Patients were diagnosed by physicians and then referred to the observers and clinical tests were repeated to ensure consistency in diagnosis. The demographic data (age, sex, dominant side, pain duration, occupational status) were recorded at the beginning of the study. The study protocol was approved by the Fatih Sultan Mehmet Training and Research Hospital Ethics Committee (approval no: KAEK 2018/36). A written informed consent was obtained from each patient. The study was conducted in accordance with the principles of the Declaration of Helsinki.

Inclusion criteria were (i) age between 18-70 years and (ii) positive results of at least three of five tests (Neer, Hawkins-Kennedy, painful arc, empty can [Jobe], and external rotation resistance). ${ }^{19}$ Exclusion criteria included (i) cervical pathology, (ii) motor or sensory dysfunction, (iii) a history of malignancy, (iv) current pregnancy, (v) diabetes mellitus, (vi) total rupture of supraspinatus tendon or adhesive capsulitis, (vii) bilateral involvement, and (viii) inflammatory disease.

The sample size was calculated using the Power Analysis and Sample Size Software 15 (2017) (NCSS, LLC., Kaysville, UT, USA; www. ncss.com/software/pass). It was revealed that a random sample of 100 subjects, each measured 
twice, produced a two-sided 95\% confidence interval (CI) with width of 0.200 when the estimated intraclass correlation is 0.700 using a one-way random-effects analysis of variance model. ${ }^{20}$

A $5-10-\mathrm{mHz}$ linear probe was used in $\mathrm{B}$ mode (Mindray Medical International Co., Ltd., Shenzhen, China). The supraspinatus tendon thickness, acromiohumeral distance, and occupation ratio were measured by two observers with at least two years of experience using musculoskeletal ultrasonography. Prior to the start of the study, both observers undertook pilot ultrasound examinations of five individuals (not included in the study) until they felt confident about achieving reproducible measurements. Preset ultrasonographic parameters for musculoskeletal imaging were selected on the ultrasound scanner, which included depth, focus point, gain, and fusion, adjusted depending on the ultrasound view and the individual being scanned, in order to achieve the best imaging of the landmarks. ${ }^{21}$

The ultrasound measurements were performed twice for each patient by two different physicians, one week apart. In each session, the patient was evaluated by two observers consecutively. All sonograms were stored in the ultrasound instrument, and the measurements were taken at a separate time from testing after completion of all measurements of all patients. During the testing and measurement sessions, the observers, who were blinded to the names of the patients, were never together in the examining room when taking the measurements from the sonogram. Therefore, each observer was blinded to the measurements of the other and his or her own previous measurements.

Supraspinatus tendon thickness measurements were performed with the patient seated upright with the feet resting flat on the floor, the trunk in a neutral position, and the head facing forward. The participants were asked to place the palm of their hand on the buttock on the affected side (Crass position). ${ }^{22}$

The supraspinatus tendon was examined in the transverse plane. The ultrasound transducer was placed on the anterior aspect of the shoulder and then moved laterally to visualize the supraspinatus tendon and then anterior to visualize both the supraspinatus tendon and the long head of the biceps tendon along the short axis. The reference point was lateral of the hyperechoic lamina of the biceps tendon. From this point, three measurements were performed along the tendon at 10, 15, and $20 \mathrm{~mm}$ (Figure 1a). The lower limit of the supraspinatus tendon was defined as the hyperechoic region over the anechoic articular cartilage of the humeral head, and the upper limit was defined as the hyperechoic region under the anechoic subdeltoid bursa. Considering the irregularity of the supraspinatus tendon structure, three different measurements were averaged to provide a single value for supraspinatus tendon thickness. ${ }^{11}$

For the acromiohumeral distance measurement, the patients were seated in a neutral position with the arms resting by the
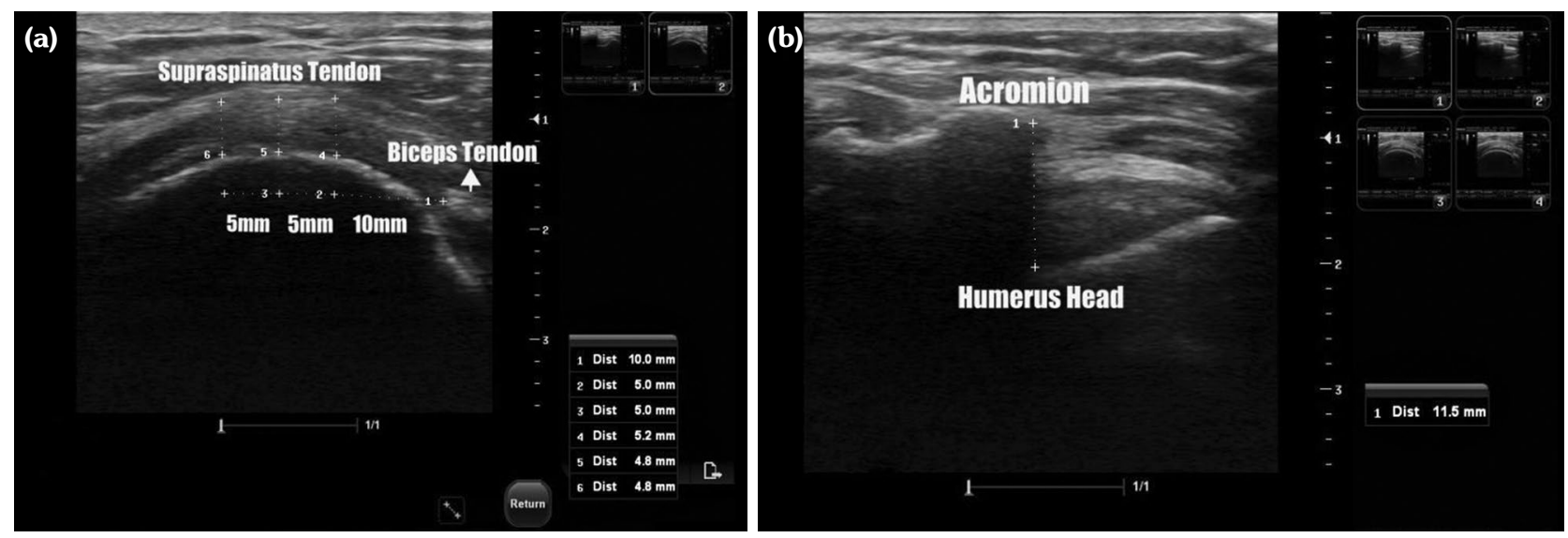

Figure 1. (a) Supraspinatus tendon thickness in transverse plane: Average of three measures taken at 10, 15, and 20 mm lateral to biceps tendon. (b) Linear measurement of acromiohumeral distance taken from acromial tip to humeral head. 
sides. The ultrasound transducer was placed longitudinally on the anterior aspect of the anterior acromial margin so that both the humeral head and acromion were visualized. The acromiohumeral distance was defined as the linear distance between the superior aspect of the humeral head and the inferior aspect of the acromion (Figure 1b). ${ }^{11}$

In the transverse view, the occupation ratio was calculated as (supraspinatus tendon thickness/acromiohumeral distance) $\times 100 .^{11}$

\section{Statistical analysis}

Normal distribution of the data was assessed using the Shapiro-Wilk test. As all data were normally distributed, the mean \pm standard deviation (SD) values were used for parametric comparisons between groups using the paired $\mathrm{t}$-test. For categorical variables, frequency and percentages were used as descriptive statistics.

In addition, the intraclass correlation coefficient (ICC), minimum detectable change (MDC), and standard error of the mean (SEM) were calculated for the supraspinatus tendon thickness. The acromiohumeral distance and occupation ratio values were used to determine the intra-rater reliability coefficient. ${ }^{23-25}$ The ICC was derived from analysis of variance. Intra- and inter-rater reliability coefficients were assessed by comparing

Table 1. Demographic parameters of participants $(n=83)$

\begin{tabular}{lccc}
\hline & $\mathrm{n}$ & $\%$ & Mean $\pm \mathrm{SD}$ \\
\hline Age (year) & & & $51.6 \pm 11.0$ \\
Body mass index $\left(\mathrm{kg} / \mathrm{m}^{2}\right)$ & & & $26.0 \pm 4.8$ \\
Sex & & & \\
$\quad$ Male & 21 & 25.3 & \\
$\quad$ Females & 62 & 74.7 & \\
Occupation & & & \\
$\quad$ Housewife & 45 & 54.2 & \\
$\quad$ Civil servant & 22 & 26.5 & \\
$\quad$ Worker & 2 & 2.4 & \\
$\quad$ Retired & 4 & 4.8 & \\
Pain duration (month) & & & $12.0 \pm 4.2$ \\
Dominant side & & & \\
$\quad$ Right & 79 & 95.2 & \\
$\quad$ Left & 4 & 4.8 & \\
Symptomatic side & & & \\
$\quad$ Right & 54 & 65.1 & \\
$\quad$ Left & 29 & 34.9 & \\
\hline SD: Standard deviation. & & & \\
\hline
\end{tabular}

the variability of the different ratings of the same subject to the total variation across all ratings and all subjects. The ICC is a measure of the homogeneity of elements within clusters and has a maximum value of 1 when there is complete homogeneity. In the current study, a two-way mixed-effects model was used to test intra-rater reliability. ${ }^{23}$ To judge the reliability of the ICC, a value of $0.5-0.75$ indicated moderate reliability, 0.75-0.90 indicated good reliability, and $>0.90$ indicated excellent reliability. ${ }^{23}$

The SEM was calculated as SD $\times \sqrt{ }(1-\mathrm{ICC})$, where $\mathrm{SD}$ is the $\mathrm{SD}$ of the data collected during T1. ${ }^{24}$ MDC was calculated as SEM $\times 1.96$, where 1.96 represents a 95\% level of confidence and multiplying by $\sqrt{ } 2$ provides additional uncertainty to compensate for different scores of measurements from two different time points. All statistical analyses of the study data were performed using the IBM SPSS Statistics for Windows, version 25.0 software (IBM Corp., Armonk, NY, USA).

\section{RESULTS}

The demographic details of the participants are shown in Table 1. The supraspinatus tendon thickness was measured within the range of 3.2-8.7 $\mathrm{mm}$ by both observers (first observer, 3.2-8.1 $\mathrm{mm}$; second observer, 3.2-8.7 $\mathrm{mm}$ ), while acromiohumeral distance was measured within the range of $5.4-18 \mathrm{~mm}$ (first observer, 5.4-17.2 $\mathrm{mm}$; second observer 8.4-18 $\mathrm{mm}$ ). The occupation ratio values obtained by both observers were within the range of 0.33-0.79 (first observer, 0.33-0.69; second observer, 0.33-0.79). All measurements are shown in Table 2.

Table 2. Ultrasonographic measurements

\begin{tabular}{lcccc}
\hline & Observer 1 & & Observer 2 \\
\cline { 2 - 2 } & Mean \pm SD & & Mean \pm SD \\
\hline SsT thickness-1 $(\mathrm{mm})$ & $6.5 \pm 0.9$ & & $6.5 \pm 0.9$ \\
SsT thickness 2 $(\mathrm{mm})$ & $6.4 \pm 0.9$ & & $6.6 \pm 0.9$ \\
AHD-1 $(\mathrm{mm})$ & $11.8 \pm 1.4$ & & $11.8 \pm 1.53$ \\
AHD-2 $(\mathrm{mm})$ & $13.2 \pm 1.3$ & & $13.19 \pm 1.26$ \\
Occupation ratio-1 & $0.5 \pm 0.1$ & & $0.54 \pm 0.09$ \\
Occupation ratio-2 & $0.5 \pm 0.1$ & & $0.55 \pm 0.09$ \\
\hline SD: Standard deviation; & SsT: & Supraspinatus & tendon; AHD: \\
Acromiohumeral distance. & & \\
\hline
\end{tabular}




\begin{tabular}{|c|c|c|c|c|}
\hline Comparison & Statistic & SsT thickness & AHD & Occupation ratio \\
\hline \multicolumn{5}{|l|}{ Intra-rater } \\
\hline \multirow{4}{*}{ Observer 1} & ICC & 0.914 & 0.943 & 0.916 \\
\hline & $95 \% \mathrm{CI}$ & $0.871-0.944$ & $0.914-0.963$ & $0.873-0.945$ \\
\hline & SEM (mm) & $0.26(4.13 \%)$ & 0.24 (2.07\%) & $0.017(3.2 \%)$ \\
\hline & $\mathrm{MDC}(\mathrm{mm})$ & 0.740 & 0.665 & 0.047 \\
\hline \multirow{4}{*}{ Observer 2} & ICC & 0.929 & 0.933 & 0.882 \\
\hline & $95 \% \mathrm{CI}$ & $0.892-0.953$ & $0.899-0.956$ & $0.824-0.923$ \\
\hline & $\mathrm{SEM}(\mathrm{mm})$ & 0.18 (2.77\%) & 0.28 (2.37\%) & $0.022(4 \%)$ \\
\hline & $\mathrm{MDC}(\mathrm{mm})$ & 0.498 & 0.770 & 0.060 \\
\hline \multirow{4}{*}{ Inter-rater } & ICC & 0.916 & 0.943 & 0.790 \\
\hline & $95 \% \mathrm{CI}$ & 0.873-0.945 & $0.914-0.963$ & $0.693-0.853$ \\
\hline & SEM (mm) & 0.19 (2.13\%) & $0.243(2.06 \%)$ & $0.028(5.18 \%)$ \\
\hline & $\mathrm{MDC}(\mathrm{mm})$ & 0.526 & 0.673 & 0.077 \\
\hline
\end{tabular}

The first observer had excellent intra-rater reliability for supraspinatus tendon thickness (ICC $=0.914 ;$ 95\% CI; 0.871-0.944; p<0.001), acromiohumeral distance measurements (ICC $=0.943 ;$ 95\% CI; 0.914-0.963; p<0.001) and occupation ratio $(\mathrm{ICC}=0.916 ; 95 \% \mathrm{CI}$; 0.873-0.945; $p<0.001$ ).

The second observer had excellent intrarater reliability for supraspinatus tendon thickness (ICC=0.929; 95\% CI; 0.892-0.953; p<0.001) and acromiohumeral distance measurements (ICC $=0.933 ;$ 95\% CI; 0.899-0.956; p<0.001). Also, the second observer had good intra-rater reliability for the occupation ratio (ICC $=0.882$; 95\% CI; 0.824-0.923; $\mathrm{p}<0.001$ ). The SEM values were low within the range of $0.0170 .26 \mathrm{~mm}$ for the first observer and $0.022-0.28 \mathrm{~mm}$ for the second. The MDC values were also found to be low within the range of $0.047-0.740 \mathrm{~mm}$ for the first observer and $0.06-0.770 \mathrm{~mm}$ for the second.

Inter-rater reliability for supraspinatus tendon thickness (ICC $=0.916 ; 95 \% \mathrm{CI} ; 0.873-0.945$; $\mathrm{p}<0.001)$ and acromiohumeral distance measurements (ICC $=0.943$; 95\% CI; 0.914-0.963; $p<0.001)$ was excellent. Inter-rater reliability reliability for the occupation ratio was good (ICC $=0.790 ; 95 \%$ CI; 0.693-0.850, $\mathrm{p}<0.001)$. The SEM values were low within the range of
0.028-0.243 mm. Values for MDC were also low within the range of $0.077-0.673 \mathrm{~mm}$. The intraand inter-rater reliability coefficients of the ICC, SEM, and MDC values are presented in Table 3.

\section{DISCUSSION}

In the present study, we evaluated the intra- and inter-rater reliability coefficients of ultrasonographic measurements of supraspinatus tendon thickness, acromiohumeral distance, and occupation ratio parameters in patients diagnosed with shoulder impingement syndrome. Excellent intra- and inter-rater reliability coefficients were achieved for supraspinatus tendon and acromiohumeral distance, consistent with the findings of previous studies. ${ }^{15,18}$

Different measurement methods have been used in previous ultrasound studies. For instance, Leong et al. $^{15}$ performed transverse imaging and calculated the average supraspinatus tendon thickness at 10, 20, and $30 \mathrm{~mm}$ lateral from the long head of the biceps tendon in cases with irregular tendon thickness. Hougs Kjæer et al. ${ }^{16}$ preferred longitudinal imaging and measured the thickness at $20 \mathrm{~mm}$ from the supraspinatus tendon insertion point on the humerus in 
two different positions, and McCreesh et al. ${ }^{18}$ performed transverse imaging and calculated the mean thickness at 5 and $10 \mathrm{~mm}$ lateral from the long head of the biceps tendon. In the current study, we reasoned that measurements from three different points would increase reliability, so the supraspinatus tendon thickness was measured at 10,15 , and $20 \mathrm{~mm}$ lateral to the biceps tendon on transverse images. ${ }^{15}$ The average of these three measurements was calculated and used for analyses, as described by Michener et al. ${ }^{11}$ Although different methods have been used in recent studies, excellent intra- and inter-rater reliability coefficients have been reported. Leong et al. ${ }^{15}$ also reported excellent intra-rater reliability on transverse images (ICC $=0.92 ; \mathrm{MDC}=0.64 \mathrm{~mm}$; $\mathrm{SEM}=0.23 \mathrm{~mm}$ ). Hougs Kjzer et al. ${ }^{16}$ reported excellent intra-rater reliability of the supraspinatus tendon thickness recorded on longitudinal views $\quad(\mathrm{ICC}=0.98-0.98 ; \mathrm{MDC}=0.47-0.59 \mathrm{~mm}$; $\mathrm{SEM}=0.12-0.15 \mathrm{~mm})$ and excellent inter-rater reliability $(\mathrm{ICC}=0.92-0.96$; $\mathrm{MDC}=0.76-0.84 \mathrm{~mm}$; $\mathrm{SEM}=0.11-0.14 \mathrm{~mm})$. McCreesh et al. ${ }^{18}$ examined supraspinatus tendon thickness in 45 patients with rotator cuff tendinopathy and reported excellent intra-rater reliability (ICC $=0.97$; $\mathrm{MDC}=0.6 \mathrm{~mm} ; \mathrm{SEM}=0.3 \mathrm{~mm})$ and interrater reliability $(\mathrm{ICC}=0.94 ; \mathrm{MDC}=1.3 \mathrm{~mm}$; $\mathrm{SEM}=0.5 \mathrm{~mm}){ }^{18}$ The measurement techniques used in the current study were similar to those employed in the previous reports.

The acromiohumeral distance is another important measurement in shoulder impingement syndrome. However, the methods used to measure it differed in recent studies. In general, the shortest distance between the acromion and the humeral head is measured, usually along a line parallel to the acoustic shadow cast by the acromion. 3,12-14,26,27 In contrast, three studies measured the distance between the edge of the acromion and the tip of the greater tuberosity, which is a longer distance anatomically. ${ }^{28-30}$ Duerr ${ }^{21}$ reported similar degrees of reliability for both measurements, although dynamic evaluation presents another issue with acromiohumeral distance measurements. Mackenzie et $a .^{20}$ reported ICC scores that were fair to good for inter-rater reliability of acromiohumeral distance measurements in all three of the shoulder positions tested (neutral, 0.88; passive arm abduction, 0.65; active arm abduction, 0.68). Desmeules et al. ${ }^{14}$ observed a more pronounced dynamic narrowing during abduction from $0^{\circ}$ to $45^{\circ}$, which might indicate that the shorter acromiohumeral distance in shoulder impingement syndrome patients could be due to increased superior translation of the humeral head caused by rotator cuff failure or pain inhibition. In this study, acromiohumeral distance was measured with the arms of the patient in a natural resting posture since it is the easiest method to standardize in the typical clinical setting.

Pijls et al. ${ }^{12}$ measured the acromiohumeral distance in the neutral position in 43 patients with shoulder impingement syndrome and reported excellent intra-rater reliability ( $\mathrm{ICC}=0.92-0.94)$ and good inter-rater reliability ( $\mathrm{ICC}=0.70)$. The authors pointed out that the interpretation of ultrasound images is less reliable in symptomatic patients because of the lack of clarity of the hyperechoic landmarks in the presence of fibrous or calcific changes. Thus, they concluded that the MDC and SEM values are much more important in studies evaluating measurements of symptomatic patients with rotator cuff tendinopathy or shoulder impingement syndrome. The low SEM and MDC values suggest that practitioner error had a minimal impact on the overall error of the measurement and that error was due to systematic variation. Therefore, one can be confident that the measurements of the different examiners were reliable. $^{17}$

McCreesh et al. ${ }^{18}$ examined acromiohumeral distance in 45 patients with rotator cuff tendinopathy using the same technique as in the current study and reported excellent intra-rater reliability $(\mathrm{ICC}=0.98 ; \quad \mathrm{MDC}=0.7$; $\mathrm{SEM}=0.3 \mathrm{~mm})$ and inter-rater reliability (ICC=0.95; MDC=1.2 $\mathrm{mm}$; SEM=0.4 mm). Likewise, Kalra et al. ${ }^{26}$ reported excellent intra-rater reliability $\quad(\mathrm{ICC}=0.76-0.92$; $\mathrm{MDC}=1.3-2.2 \mathrm{~mm} ; \mathrm{SEM}=0.9-1.6 \mathrm{~mm}$ ) in 31 patients with rotator cuff tendinopathy. Moreover, Hougs Kjær et al. ${ }^{16}$ examined 22 patients with shoulder impingement syndrome and reported excellent intra-rater reliability $\quad$ (ICC $=0.96$; $\mathrm{MDC}=0.98 \mathrm{~mm}$; $\mathrm{SEM}=0.19 \mathrm{~mm})$ and interrater reliability $(\mathrm{ICC}=0.96 ; \mathrm{MDC}=1.06 \mathrm{~mm}$; $\mathrm{SEM}=0.20 \mathrm{~mm}$ ). In the present study, similar to recent reports, intra- and inter-rater reliability were excellent with ICC $>0.90$, low MDC 
(0.6 and $1.06 \mathrm{~mm}$, respectively), and low SEM ( 0.3 and $0.24 \mathrm{~mm}$, respectively).

To our knowledge, this is the first study to evaluate the intra- and inter-rater reliability of the occupation ratio. However, its reliability should be predictable, according to the results of other studies reporting high reliability for supraspinatus tendon and acromiohumeral distance. In our study, the intra-rater reliability for occupation ratio was excellent for the first observer and good for the second, while the inter-rater reliability was good for both.

Michener et al. ${ }^{11}$ used the occupation ratio for the first time to define the relationship between supraspinatus tendon thickness and the acromiohumeral distance in patients with shoulder impingement syndrome in order to elucidate the potential extrinsic mechanism. Supraspinatus tendon thickness was reported as $6 \mathrm{~mm}$ and the acromiohumeral distance as $10.8 \mathrm{~mm}$ with the arms at the side, leaving $4.2 \mathrm{~mm}$ of the acromiohumeral distance unoccupied by the tendon. The authors suggested that extrinsic compression of the tendon could occur with arm elevation, although the shoulder was not imaged during dynamic motion to determine whether impingement of the tendon had actually occurred. The occupation ratio was calculated as $61.7 \pm 10.3 \%$ in the patient group and $54.2 \pm 7.9 \%$ in the control group. In the current study, the occupation ratio was lower than that reported by Michener et al. ${ }^{31}$ (first observer, $0.5 \pm 0.1 \%$; second observer, $0.5 \pm 0.1 \%$ ), with a good ICC value of 0.077 . When the shoulder is evaluated at $60^{\circ}$ abduction, acromiohumeral distance is known to decrease by $1.6-4.2 \mathrm{~mm}$, as compared to the neutral position, suggesting that external mechanisms can be activated at $60^{\circ}$ abduction; thus, the occupation ratio should be measured at $60^{\circ}$. As previously stated, the acromiohumeral distance measurement in the neutral position is considered to be the easiest way to achieve standardization in the typical clinical setting, although for occupation ratio measurements, dynamic evaluation would be more appropriate.

As a limitation to this study, acromiohumeral distance was not measured during shoulder abduction. Hence, further studies are needed to evaluate the dynamic occupation ratio parameter in a larger population with a healthy control group. A second limitation was the lack of an overall agreement established before commencing with the study.

Despite these limitations, the results of the present study are expected to contribute to clinical practice. Previous studies have demonstrated a relationship between the acromiohumeral distance and shoulder function in shoulder impingement syndrome patients. ${ }^{14,32}$ In one study, pain, daily living activities, shoulder range of motion, and muscle strength were all correlated to the acromiohumeral distance in patients with shoulder impingement syndrome without rotator cuff tears. ${ }^{32}$ Therefore, measuring the supraspinatus dimension, acromiohumeral distance, and supraspinatus tendon thickness in proportion to the subacromial space may aid clinicians in understanding the source of the problem, treatment planning, treatment follow-up, and decisions for surgical or conservative treatment options.

In conclusion, the results of this study showed that the acromiohumeral distance, supraspinatus tendon thickness, and occupation ratio in patients with symptomatic shoulder impingement syndrome can be reliably measured on ultrasound images by either a single examiner or a pair of examiners, and the MDC and SEM values provided are useful parameters for future studies. Further studies are warranted to determine the importance of ultrasound imaging of patients with subacromial impingement to establish a definite diagnosis, monitor treatment, and predict the treatment outcome.

\section{Declaration of conflicting interests}

The authors declared no conflicts of interest with respect to the authorship and/or publication of this article.

\section{Funding}

The authors received no financial support for the research and/or authorship of this article.

\section{REFERENCES}

1. Michener LA, McClure PW, Karduna AR. Anatomical and biomechanical mechanisms of subacromial impingement syndrome. Clin Biomech (Bristol, Avon) 2003;18:369-79. 
2. Neer CS 2nd. Anterior acromioplasty for the chronic impingement syndrome in the shoulder: a preliminary report. J Bone Joint Surg [Am] 1972;54:41-50.

3. Seitz AL, Michener LA. Ultrasonographic measures of subacromial space in patients with rotator cuff disease: A systematic review. J Clin Ultrasound 2011;39:146-54.

4. McCreesh KM, Crotty JM, Lewis JS. Acromiohumeral distance measurement in rotator cuff tendinopathy: is there a reliable, clinically applicable method? A systematic review. Br J Sports Med 2015;49:298-305.

5. Cholewinski JJ, Kusz DJ, Wojciechowski P, Cielinski LS, Zoladz MP. Ultrasound measurement of rotator cuff thickness and acromio-humeral distance in the diagnosis of subacromial impingement syndrome of the shoulder. Knee Surg Sports Traumatol Arthrosc 2008;16:408-14.

6. Joensen J, Couppe C, Bjordal JM. Increased palpation tenderness and muscle strength deficit in the prediction of tendon hypertrophy in symptomatic unilateral shoulder tendinopathy: an ultrasonographic study. Physiotherapy 2009;95:83-93.

7. Wu $\mathrm{CH}$, Wang $\mathrm{YC}$, Wang $\mathrm{HK}$, Chen WS, Wang TG. Evaluating displacement of the coracoacromial ligament in painful shoulders of overhead athletes through dynamic ultrasonographic examination. Arch Phys Med Rehabil 2010;91:278-82.

8. Wu $\mathrm{CH}$, Chang $\mathrm{KV}$, Su PH, Kuo WH, Chen WS, Wang TG. Dynamic ultrasonography to evaluate coracoacromial ligament displacement during motion in shoulders with supraspinatus tendon tears. J Orthop Res 2012;30:1430-4.

9. Diercks R, Bron C, Dorrestijn O, Meskers C, Naber R, de Ruiter $\mathrm{T}$, et al. Guideline for diagnosis and treatment of subacromial pain syndrome: a multidisciplinary review by the Dutch Orthopaedic Association. Acta Orthop 2014;85:314-22.

10. Roy JS, Braën C, Leblond J, Desmeules F, Dionne CE, MacDermid JC, et al. Diagnostic accuracy of ultrasonography, MRI and MR arthrography in the characterisation of rotator cuff disorders: a systematic review and meta-analysis. $\mathrm{Br} \mathrm{J}$ Sports Med 2015;49:1316-28.

11. Michener LA, Subasi Yesilyaprak SS, Seitz AL, Timmons MK, Walsworth MK. Supraspinatus tendon and subacromial space parameters measured on ultrasonographic imaging in subacromial impingement syndrome. Knee Surg Sports Traumatol Arthrosc 2015;23:363-9.

12. Pijls BG, Kok FP, Penning LI, Guldemond NA, Arens HJ. Reliability study of the sonographic measurement of the acromiohumeral distance in symptomatic patients. J Clin Ultrasound 2010;38:128-34.

13. White CE, Dedrick GS, Apte GG, Sizer PS, Brismée $\mathrm{JM}$. The effect of isometric shoulder internal and external rotation on the acromiohumeral distance. Am J Phys Med Rehabil 2012;91:193-9.
14. Desmeules F, Minville L, Riederer B, Côté CH, Frémont P. Acromio-humeral distance variation measured by ultrasonography and its association with the outcome of rehabilitation for shoulder impingement syndrome. Clin J Sport Med 2004;14:197-205.

15. Leong HT, Tsui S, Ying M, Leung VY, Fu SN. Ultrasound measurements on acromio-humeral distance and supraspinatus tendon thickness: test-retest reliability and correlations with shoulder rotational strengths. J Sci Med Sport 2012;15:284-91.

16. Hougs Kjæer B, Ellegaard K, Wieland I, Warming S, Juul-Kristensen B. Intra-rater and inter-rater reliability of the standardized ultrasound protocol for assessing subacromial structures. Physiother Theory Pract 2017;33:398-409.

17. Mackenzie TA, Herrington L, Horlsey I, Cools A. An evidence-based review of current perceptions with regard to the subacromial space in shoulder impingement syndromes: Is it important and what influences it? Clin Biomech (Bristol, Avon) 2015;30:641-8.

18. McCreesh KM, Anjum S, Crotty JM, Lewis JS. Ultrasound measures of supraspinatus tendon thickness and acromiohumeral distance in rotator cuff tendinopathy are reliable. $\mathrm{J}$ Clin Ultrasound 2016;44:159-66.

19. Alqunaee M, Galvin R, Fahey T. Diagnostic accuracy of clinical tests for subacromial impingement syndrome: a systematic review and meta-analysis. Arch Phys Med Rehabil 2012;93:229-36.

20. Mackenzie TA, Bdaiwi AH, Herrington L, Cools A. Inter-rater Reliability of Real-Time Ultrasound to Measure Acromiohumeral Distance. PM R 2016;8:629-34.

21. Duerr M. Reliability and Accuracy of Distance Measurements Between Shoulder Bony Landmarks Evaluated by Ultrasound in Asymptomatic Subjects [Master's Thesis]. New Zealand: Auckland University of Technology; 2010.

22. Crass JR, Craig EV, Feinberg SB. The hyperextended internal rotation view in rotator cuff ultrasonography. $\mathrm{J}$ Clin Ultrasound 1987;15:416-20.

23. Shrout PE, Fleiss JL. Intraclass correlations: uses in assessing rater reliability. Psychol Bull 1979;86:420-8.

24. Bruton A, Conway JH, Holgate ST. Reliability: what is it, and how is it measured? Physiotherapy 2000;86:94-9.

25. Eliasziw M, Young SL, Woodbury MG, FrydayField K. Statistical methodology for the concurrent assessment of interrater and intrarater reliability: using goniometric measurements as an example. Phys Ther 1994;74:777-88.

26. Kalra N, Seitz AL, Boardman ND 3rd, Michener LA. Effect of posture on acromiohumeral distance with arm elevation in subjects with and without rotator cuff disease using ultrasonography. J Orthop Sports Phys Ther 2010;40:633-40. 
27. Maenhout A, van Cingel R, De Mey K, Van Herzeele $\mathrm{M}$, Dhooge F, Cools A. Sonographic evaluation of the acromiohumeral distance in elite and recreational female overhead athletes. Clin J Sport Med 2013;23:178-83.

28. Schmidt WA, Schmidt H, Schicke B, Gromnica-Ihle E. Standard reference values for musculoskeletal ultrasonography. Ann Rheum Dis 2004;63:988-94.

29. Wang HK, Lin JJ, Pan SL, Wang TG. Sonographic evaluations in elite college baseball athletes. Scand J Med Sci Sports 2005;15:29-35.

30. Kumar P, Bradley M, Swinkels A. Within-day and day-to-day intrarater reliability of ultrasonographic measurements of acromion-greater tuberosity distance in healthy people. Physiother Theory Pract 2010;26:347-51.

31. Malanga GA, Chu SK, Ramirez Del Toro J, Karnaugh RD, Dentico R, Komaroff E. Sonographic evaluation of supraspinatus cross-sectional area in collegiate baseball players. PM R 2012;4:488-92.

32. Mayerhoefer ME, Breitenseher MJ, Wurnig C, Roposch A. Shoulder impingement: relationship of clinical symptoms and imaging criteria. Clin J Sport Med 2009;19:83-9. 